\title{
Studies of stellar evolution using masers
}

\author{
Anna Bartkiewicz* \\ Centre for Astronomy, Faculty of Physics, Astronomy and Informatics, Nicolaus Copernicus \\ University, Grudziadzka 5, 87-100 Torun, Poland, \\ E-mail: annan@astro.umk.pl
}

\begin{abstract}
High angular resolution studies of maser emisson at radio and $\mathrm{mm}$ wavelengths provide one of the best existing tools for deriving kinematics and physical conditions of regions that are hidden in the dense environments and are not reachable at other wavelength ranges. Therefore, the European VLBI Network, as well as other VLBI networks, are successfully used to investigate high-mass star forming regions and evolved stars. With the milliarcsecond resolution and sensitivity of a few mJy, VLBI provides detailed information concerning proper motions and magnetic field. This is a summary of recent observational progress on masers at $\mathrm{cm}$ wavelengths and how these results have affected our understanding of stellar evolution. While this summary will utilize some of the most characteristic, recent maser results to illustrate the findings, it is obviously not possible to cover all ongoing research in this proceeding. Perhaps this reflects the large amount of activity in our maser community.
\end{abstract}

14th European VLBI Network Symposium \& Users Meeting (EVN 2018)

8-11 October 2018

Granada, Spain

${ }^{*}$ Speaker. 


\section{Introduction}

Maser action, i.e., microwave amplification by stimulated emission radiation, occurs naturally in interstellar space under non-LTE conditions, velocity coherence is achieved between a group of population-inverted molecules [14]. Maser emission, mostly from $\mathrm{OH}, \mathrm{H}_{2} \mathrm{O}, \mathrm{CH}_{3} \mathrm{OH}$ and $\mathrm{SiO}$, appears in regions close to newly born high-mass and low-mass stars, as well as in the envelopes of evolved stars. The combination of masers and Very Long Baseline Interferometry (VLBI) allows a unique capability of studying these densely obscured regions in detail. For example, a recent Australia Telescope Compact Array (ATCA) survey of the $1.6 \mathrm{GHz}$ OH maser transition identified 122 (57\% of the sample) sites associated with evolved stars (one of which is a planetary nebula), $64(30 \%)$ with star formation, two sites with supernova remnants, and 27 (13\%) of unknown origin [27].

Several of the most recent results related to maser research were presented at the International Astronomical Union Symposium No. 336 in Cagliari, Sardinia [2]. Out of 105 proceedings, 39 presented results obtained using VLBI and nine of which were obtained using the European VLBI Network (EVN). EVN has played an especially important role in the $6.7 \mathrm{GHz}$ methanol maser science. For example, the morphology of such emission in high-mass star-forming regions (HMSFRs) was widely studied; using 8-10 radio telescopes a diversity of structures has been investigated in detail, revealing a wide diversity of maser morphologies including simple emission regions, arcs, rings and more complex structures [4],[5]. These studies were made possible due to the high sensitivity of a few mJy per beam in a single channel, and the high angular resolution of a few milliarcseconds. For such observations the narrow $6.7 \mathrm{GHz}$ methanol maser lines require a very high spectral resolution, where typically a $2 \mathrm{MHz}$ bandwidth must be divided into 1024 (or 2048) spectral channels to yield $90 \mathrm{~m} \mathrm{~s}^{-1}$ ( or $45 \mathrm{~m} \mathrm{~s}^{-1}$ ) velocity resolution.

\section{2. "Maser news" in high-mass star-forming regions}

In recent years, the VLBI technique has enabled $6.7 \mathrm{GHz}$ methanol maser observations to determine milliarcsecond scale gas kinematics in HMSFRs. A planar infall of methanol gas around Cepheus A HW2 was registered, implying the existence of an accretion disk [33]. EVN multiepoch observations over two years showed that the three-dimensional velocity field contains a contraction velocity of approximately $2 \mathrm{~km} \mathrm{~s}^{-1}$, at distances from HW2 between 300 to 900 AU. In turn, this implies an accretion rate of the order of $10^{-4} \mathrm{M}_{\odot} \mathrm{yr}^{-1}$ at a distance of $300 \mathrm{AU}$ from the star. At distances closer to the star, below at radii less than $300 \mathrm{AU}$, a rotational component of around $4 \mathrm{~km} \mathrm{~s}^{-1}$ was measured. Another interesting result with very detailed picture of the gas dynamics was presented in a massive YSO G023.01-00.41 [31]. The magnetic field configuration within a radius of $2000 \mathrm{AU}$ from the central source was studied using the EVN in full polarization mode. The recent quantitative, theoretical model of the magnetic properties of methanol solved "an important puzzle in astrochemistry - how to measure magnetic fields in space using methanol, the simplest form of alcohol" [19]. That includes the complicated hyperfine structure that results from its internal rotation and new calculations of Landé g-factors for all the hyperfine transitions of the methanol molecule. 
The VLBI Exploration of Radio Astrometry interferometer (VERA) is widely used as a distance estimator to maser sources and studying their kinematics. An example is the HMSFR AFGL 5142 with a $2.14 \mathrm{kpc}( \pm 2 \%)$ distance measured via the trigonometric parallax method and the same data reveal remarkable bipolar bowshocks expanding from the most massive member [8].

Quite often the results of maser emission imaging stimulate further studies of selected regions. Some of the most basic questions we try to answer are: What is inside and nearby of the maser?, Where is a driving source?. The above mentioned HMSFR G23.01-0.41 was first known as showing an outflow combined with a rotation owing to the multi-epoch EVN imaging of $6.7 \mathrm{GHz}$ methanol emission [29]. Follow-up observations using SMA provided subarcsecond maps of various molecular lines, including both hot-core and outflow tracers [30]. Next, searches using VLA and ALMA of 22, 45 and $200 \mathrm{GHz}$ continuum allowed the discovery of a collimated thermal jet [32] , and a rotating molecular disk undergoing infall toward the central star was imaged directly [35]. The methanol masers are likely originating from deep inside this region, tracing the inner outflow cavity.

In many cases maser emission helps to unveil information from the interiors of dense regions. In NGC 6334I, an outburst of (sub)millimeter continuum in the protocluster MM1 was detected, along with multi-frequency maser emission from different species as water and methanol [7], [16]. Only some continuum sources are related to masers.

As mentioned briefly above, masers are useful for magnetic field estimates. The magnetic field strength can be measured via the Zeeman splitting, when observed. Recently, it has been shown that the $44 \mathrm{GHz}$ Class I methanol maser line can be used for such studies. Towards DR21(OH) Stokes I and V profiles obtained using VLA clearly showed the Zeeman splitting, implying a field strength of about $50 \mathrm{mG}$ [24].

Further, sensitive VLA studies have also proven crucial for finding shocked gas showing maser emission in only one of the $\mathrm{OH}$ hyperfine L-band transitions, the $1720 \mathrm{MHz}$ line. In Sgr B2(M), a part of the well known star-forming molecular cloud, the $1720 \mathrm{MHz} \mathrm{OH}$ maser has been found to coincide with a $150 \mathrm{MHz}$ nonthermal radio source [42]. This implies either the site of a SNR molecular gas interaction, or a wind-wind collision in a massive binary system.

Systematic searches for new masers or consistent, multifrequency surveys are crucial to answer the general questions: How, where and when are the maser lines being formed? In recent years, especially surveys using ATCA, have been done. They were dedicated to study the associations of the 6035 and $6030 \mathrm{MHz} \mathrm{OH}$ and $6668 \mathrm{MHz}$ methanol masers [3], to compare the occurrence of 6.7 GHz and $12.2 \mathrm{GHz}$ methanol masers with $22 \mathrm{GHz}$ water masers and $6035 \mathrm{MHz}$ excited-state $\mathrm{OH}$ masers [6], to study for highly-excited methanol maser lines Class I and II [12], [21]. Two new projects have been announced recently:

1) POETS (Protostellar Outflows at the EarliesT Stages), aiming to study the dynamical properties of the outflow emission in the vicinity of luminous young stars [36],

2) KaVA (Korean and VERA Array), aiming to perform a systematic observational study of the $22 \mathrm{GHz}$ water masers and $44 \mathrm{GHz}$ class I methanol masers in HMSFRs followed by JVN and ALMA observations [18]. 


\section{Masers in evolved stars}

Maser emission is used to study the dense regions surrounding evolved stars. Using the Yebes radio telescope, single-dish monitoring of the $43 \mathrm{GHz} \mathrm{SiO}$ emission from evolved stars suggests that the emission comes from the innermost circumstellar layers [43]. More VLBI observations would be helpful to clarify details, however, these are challenging due to a large amount of flux density being resolved out [9]. Observations using the IRAM telescope revealed that $86 \mathrm{GHz} \mathrm{SiO}$ masers are common in O-rich AGB stars [23]. This illustrates the need of having the high-frequency network.

$\mathrm{OH}$ and water maser observations towards the Mira star o Ceti with the Nançay, Medicina and Effelsberg radio telescopes, in addition to MERLIN and EVN-(e)MERLIN, showed that the flaring regions are found to be less than $40 \pm 4 \mathrm{AU}$ and, most unusually, the $\mathrm{OH}$ maser zone coincides with the water maser zone. The magnetic fields in the circumstellar envelope are found to be important, as can be seen from the highly ordered linear polarization vectors observed in the $\mathrm{OH}$ maser components of o Ceti [13].

Multi-epoch VLBA observations allowed the imaging of a remarkable $22 \mathrm{GHz}$ water fountain at post-AGB star IRAS 18113-2503 with spectacular bipolar bow shocks in their high-speed, collimated, jet-driven outflows. The jets are formed in very short-lived, episodic outbursts, which may indicate episodic accretion in an underlying binary system [25]. Another interesting work has been done in the circumstellar envelope of the post-AGB star nebula IRAS 18043-2116 using ATCA, JVLA and VLT. The high-velocity water maser spectral features and the shock-excited $\mathrm{H}_{2}$ emission could be produced in molecular layers around high-velocity outflows [26].

\section{Variability studies}

It has been proved that maser emission is quite often variable including periodic variability [41]. In the above mentioned HMSFR NGC 6334I all maser lines are flaring: $\mathrm{OH}$, methanol and water [20]. The shortest periodic (a period of 23.9 days) and flaring flux variability of a methanol maser at $6.7 \mathrm{GHz}$ were reported in G014.23-00.50 [38]. Even small radio telescopes are useful in variability studies, as demonstrated by the monitoring of the $22 \mathrm{GHz}$ water maser line in the semiregular variable R Crt, carried out using the Kagoshima $6 \mathrm{~m}$ telescope [37]. The velocity variation of the maser is likely caused by the shock propagation in the envelope due to stellar pulsation. Special attention should be given to multi-frequency monitoring, including emission of the central source at the infra-red bands. For the first time a relationship between the maser and infra-red variability has been established for the intermediate mass young stellar object G107.298+5.639, where the water and methanol masers show periodic and anticorrelated variability [40]. The infrared light curve exctracted from NEOWISE data shows the same period of ca. 34.6 days as the masers, but its shape is distinct from that of the maser flares [39].

Multi-epoch monitoring programs focusing on the variability of $6 \mathrm{~cm}$ formaldehyde $\left(\mathrm{H}_{2} \mathrm{CO}\right)$ masers in NGC 7538 IRS 1 from 2008 to 2015 were conducted with the GBT, WSRT, and the VLA. Also the $12.2 \mathrm{GHz}$ methanol and $22.2 \mathrm{GHz}$ water masers were observed, with a noticeable decrease in the flux. The variability may be caused by changes in the maser amplification path in regions with similar morphology and kinematics [1]. 


\section{News in the astrophysical maser science}

Here, instead of a summary, I list the maser news from recent time that are not related the topics presented in the previous sections.

- The first detection of a THz water maser in NGC7538-IRS1 with SOFIA [15],

- More methanol masers are found to be related to low-mass star-forming regions [28], [17],

- Hydrogen cyanide (HCN) masers appear to be common in carbon-rich AGB stars [22],

- A cyanoacetylene $\left(\mathrm{HC}_{3} \mathrm{~N}\right)$ maser was detected in the NGC 253 galaxy. This emission appears to be associated with a region of the galaxy close to the inner edge of the bar, where there is a significant abundance of molecular gas and widespread low-velocity shocks [10]. Class I methanol masers are located towards the edges of the nuclear molecular gas, "at the end of the bar", in regions where there is ionising radiation to dissociating the molecules. The masers arise due to large-scale cloud-cloud collisions [11].

- Masers are crucial in astrometry. A recent achievement in the precise measurements is the distance obtained via trigonometric parallax to a star-forming region on the opposite side of our Milky Way Galaxy from the Sun: $20.4 \mathrm{kpc}$ with $10 \%$ accuracy[34].

As we all can see, masers are just amazing!

\section{Acknowledgements}

I appreciate discussions and consultations with Prof. Ylva Pihlström (UNM). I acknowledge support from the National Science Centre, Poland through grant 2016/21/B/ST9/01455 and the European Union's Horizon 2020 research and innovation programme under grant agreement No 730562 [RadioNet].

\section{References}

[1] N. Andreev, E.D. Araya, I.M. Hoffman, et al., Long-term Variability of $\mathrm{H}_{2} \mathrm{CO}$ Masers in Star-forming Regions, ApJSS, 2017, 232, 29

[2] Astrophysical Masers: Unlocking the Mysteries of the Universe, eds. A. Tarchi, M.J. Reid \& P. Castangia, Proceedings of the International Astronomical Union, IAU Symposium, Cambridge University Press, 2018, 336

[3] A. Avison, L.J. Quinn, G.A. Fuller, et al., Excited-state hydroxyl maser catalogue from the methanol multibeam survey - I. Positions and variability MNRAS, 2016, 461, 136

[4] A. Bartkiewicz, M. Szymczak, H.J. van Langevelde, A.M.S. Richards and Y.M. Pihlström, The diversity of methanol maser morphologies from VLBI observations, A\&A, 2009, 502, 155

[5] A. Bartkiewicz, M. Szymczak and H.J. van Langevelde, European VLBI Network imaging of $6.7 \mathrm{GHz}$ methanol masers, A\&A, 2016, 587, A104

[6] S.L. Breen, Y. Contreras, S.P. Ellingsen, et al., The 6-GHz Multibeam Maser Survey - III. Comparison between the MMB and HOPS, MNRAS, 2018, 474, 3898 
[7] C.L. Brogan, T.R. Hunter, C.J. Cyganowski, et al., The Extraordinary Outburst in the Massive Protostellar System NGC 6334I-MM1: Flaring of the Water Masers in a North-South Bipolar Outflow Driven by $M M 1 B$, ApJ, 2018, 866, 87

[8] R.A. Burns, T. Handa, H. Imai, et al., Trigonometric distance and proper motions of $\mathrm{H}_{2} \mathrm{O}$ maser bowshocks in AFGL 5142, MNRAS, 2017, 467, 2367

[9] J.-F. Desmurs, J. Alcolea, V. Bujarrabal, F. Colomer and R. Soria-Ruiz, Missing flux in VLBI observations of $\mathrm{SiO}$ maser at $7 \mathrm{~mm}$ in IRC+10011, Proceedings of the International Astronomical Union, IAU Symposium, Cambridge University Press, 2018, 336, 387

[10] S.P. Ellingsen, X. Chen, S.L. Breen and H.-H. Qiao, Detection of $\mathrm{HC}_{3} N$ Maser Emission in NGC 253, ApJL, 2017, 841, L14

[11] S.P. Ellingsen, X. Chen, S.L. Breen and H.-H. Qiao, Class I methanol masers in NGC 253: Alcohol at the end of the bar, MNRAS, 2017, 472, 604

[12] S.P. Ellingsen, M.A. Voronkov, S.L. Breen, J.L. Caswell and A.M. Sobolev, The first high-resolution observations of 37.7-, 38.3-, and 38.5-GHz methanol masers, MNRAS, 2018, 480, 4851

[13] S. Etoka, E. Gérard, A.M.S. Richards, D. Engels, J. Brand and T. Le Bertre, Recurring OH Flares towards o Ceti - I. Location and structure of the 1990s' and 2010s' events, MNRAS, 2017, 468, 1703

[14] M.D. Gray, Maser Sources in Astrophysics, Cambridge, UK: Cambridge University Press, 2012

[15] F. Herpin, A. Baudry, A.M.S. Richards, et al., First detection of a THz water maser in NGC 7538-IRS1 with SOFIA and new 22 GHz e-MERLIN maps, A\&A, 2017, 606, A52

[16] T.R. Hunter, C.L. Brogan, G.C. MacLeod, et al., The Extraordinary Outburst in the Massive Protostellar System NGC 6334I-MM1: Emergence of Strong 6.7 GHz Methanol Masers, ApJ, 2018, 854, 170

[17] S. Kalenskii, S. Kurtz, P. Hofner, P. Bergman, C.M. Walmsley and P. Golysheva, Class I methanol masers in low-mass star formation regions, Proceedings of the International Astronomical Union, IAU Symposium, Cambridge University Press, 2018, 336, 33

[18] K.-T. Kim, T. Hirota, K. Sugiyama, et al., Understanding high-mass star formation through KaVA observations of water and methanol masers, Proceedings of the International Astronomical Union, IAU Symposium, Cambridge University Press, 2018, 336, 259

[19] B. Lankhaar, W. Vlemmings, G. Surcis, H.J. van Langevelde, G.C. Groenenboom and A. van der Avoird Characterization of methanol as a magnetic field tracer in star-forming regions, Nature Astronomy, 2018, 2, 145

[20] G.C. MacLeod, D.P. Smits, S. Goedhart, et al., A masing event in NGC 6334I: contemporaneous flaring of hydroxyl, methanol, and water masers, MNRAS, 2018, 478, 1077

[21] T.P. McCarthy, S.P. Ellingsen, M.A. Voronkov and G. Cimò, The relationship between Class I and Class II methanol masers at high angular resolution, MNRAS, 2018, 477, 507

[22] K.M. Menten, F. Wyrowski, D. Keller and T. Kamiński, Widespread HCN maser emission in carbon-rich evolved stars, A\&A, 2018, 613, A49

[23] M. Messineo, H.J. Habing, L.O. Sjouwerman, A. Omont and K.M. Menten, 86 GHz SiO maser survey of late-type stars in the Inner Galaxy. IV. SiO emission and infrared data (...), A\&A, 2018, 619, A35

[24] E. Momjian and A.P. Sarma, The Zeeman Effect in the 44 GHz Class I Methanol Maser Line toward DR21(OH), ApJ, 2017, 834, 168 
[25] G. Orosz, J.F. Gómez, H. Imai, et al., Rapidly evolving episodic outflow in IRAS 18113-2503: clues to the ejection mechanism of the fastest water fountain, MNRAS, 2019, 482, L40

[26] A.F. Pérez-Sánchez, D. Tafoya, R. García López, W. Vlemmings and L.F. Rodríguez, Deep into the Water Fountains. The case of IRAS 18043-2116 A\&A, 2017, 601, A68

[27] H.-H. Qiao, A.J. Walsh, J.A. Green, et al., Accurate OH maser positions from the SPLASH pilot region, ApJSS, 2016, 227, 26

[28] C.B. Rodríguez-Garza, S.E. Kurtz, A.I. Gómez-Ruiz, P. Hofner, E.D. Araya and S.V. Kalenskii, $A$ Catalog of $44 \mathrm{GHz}$ Methanol Masers in Massive Star-forming Regions. IV. The High-mass Protostellar Object Sample, ApJSS, 2017, 233, 4

[29] A. Sanna, L. Moscadelli, R. Cesaroni, A. Tarchi, R.S. Furuya and C. Goddi, VLBI study of maser kinematics in high-mass star-forming regions. II. G23.01-0.41 A\&A, 2010, 517, A78

[30] A. Sanna, L. Moscadelli, R. Cesaroni, et al., A subarcsecond study of the hot molecular core in G023.01-00.41, A\&A, 2014, 565, A34

[31] A. Sanna, G. Surcis, L. Moscadelli, et al., Velocity and magnetic fields within 1000 AU of a massive $Y S O$, A\&A, 2015, 583, L3

[32] A. Sanna, L. Moscadelli, R. Cesaroni, A. Caratti o Garatti, C. Goddi and C. Carrasco-González, Momentum-driven outflow emission from an O-type YSO. Comparing the radio jet with the molecular outflow, A\&A, 2016, 596, L2

[33] A. Sanna, L. Moscadelli, G. Surcis, H.J. van Langevelde, K.J.E. Torstensson and A.M. Sobolev, Planar infall of $\mathrm{CH}_{3} \mathrm{OH}$ gas around Cepheus A HW2, A\&A, 2017, 603, A94

[34] A. Sanna, M.J. Reid, T.M. Dame, K.M. Menten and A. Brunthaler, Mapping spiral structure on the far side of the Milky Way, Science, 2017, 358, 227

[35] A. Sanna, A. Kölligan, L. Moscadelli, et al., Discovery of an infalling disk with jet around a $20 M_{\odot}$ young star ALMA observations of G023.01-00.41, arXiv:1805.09842

[36] A. Sanna, L. Moscadelli, C. Goddi, V. Krishnan and F. Massi, Protostellar Outflows at the EarliesT Stages (POETS). I. Radio thermal jets at high resolution nearby $\mathrm{H}_{2} \mathrm{O}$ maser sources, A\&A, 2018, 619, A107

[37] H. Sudou, M. Shiga, T. Omodaka, C. Nakai, K. Ueda and H. Takaba, Time Variations of the Radial Velocity of H2O Masers in the Semi-Regular Variable R Crt, JKAS, 2017, 50, 157

[38] K. Sugiyama, K. Nagase, Y. Yonekura, et al., The shortest periodic and flaring flux variability of a methanol maser emission at $6.7 \mathrm{GHz}$ in G014.23-00.50, PASJ, 2017, 69, 59

[39] B. Stecklum, A. Caratti o Garatti, K. Hodapp, H. Linz, L. Moscadelli and A. Sanna, Infrared variability, maser activity, and accretion of massive young stellar objects, Proceedings of the International Astronomical Union, IAU Symposium, Cambridge University Press, 2018, 336, 37

[40] M. Szymczak, M. Olech, P. Wolak, A. Bartkiewicz and M. Gawroński, Discovery of periodic and alternating flares of the methanol and water masers in G107.298+5.639, MNRAS, 2016, 459, L56

[41] M. Szymczak, M. Olech, R. Sarniak, P. Wolak and A. Bartkiewicz, Monitoring observations of 6.7 GHz methanol masers, MNRAS, 2018, 474, 219

[42] F. Yusef-Zadeh, W. Cotton, M. Wardle and H. Intema, An OH(1720 MHz) Maser and a Nonthermal Radio Source in Sgr B2(M): An SNR-Molecular Cloud Interaction Site?, ApJL, 2016, 819, L35

[43] P. de Vicente, V. Bujarrabal, A. Díaz-Pulido, et al., ${ }^{28} \mathrm{SiO} v=0 \mathrm{~J}=1-0$ emission from evolved stars, A\&A, 2016, 589, A74 